Pure and Applied Mathematics Quarterly

Volume 7, Number 4

(Special Issue:

In memory of Eckart Viehweg)

$1241-1264,2011$

\title{
A P-adic Simpson Correspondence II: Small Representations
}

\author{
Gerd Faltings
}

\begin{abstract}
We partially extend our previous theory relating Galoisrepresentations and Higgs-bundles from Hodge-Tate to crystalline, that is we introduce Frobenius.
\end{abstract}

Keywords: p-adic Hodge theory, Frobenius bundles.

\section{INTRODUCTION}

In a previous paper ([6]) we introduced a $p$-adic Simpson correspondence, between Higgs-bundles and (generalised) étale sheaves on a curve $X$ over a $p$-adic base $\operatorname{Spec}(V)$. In Fontaine's dictionary between étale and crystalline objects this corresponds to the Hodge-Tate picture. The method was a rather straightforward application of "almost mathematics", except that at one stage we had to introduce an exponential function on $p$-adics which involved making arbitrary choices. This was necessary to treat "big" Galois-representations, that is representations which are not close to the trivial representation.

Here we try to extend this to the full crystalline theory. That is we want to associate to $\mathbb{Q}_{p}$-representations of the geometric fundamental group certain "bundles with singular connection" which are Frobenius invariant. Compared to [6] the main new ingredient is the role of Frobenius. However as there is no obvious Frobenius action on Higgs-bundles this looks like an interesting problem.

Received: Sept. 28, 2010.

1991 Mathematics Subject Classification. 14F30. 
We propose to pass from Fontaine's ring $A_{\text {cris }}$ to the more involved rings of Berger and Kedlaya. These are coordinate rings of coverings of the $p$-adic unit disk. While $A_{\text {cris }}$ corresponds roughly to power series converging for $p$-adic valuations $\geq 1 /(p-1)$, we instead consider convergence for valuations between rational numbers $b^{-1}$ and $a^{-1}$ or for valuations $\leq a^{-1}$. The action of Frobenius multiplies $a$ and $b$ by $p$, and Frobenius modules are defined via an isomorphism on the overlap of two annuli. In $A_{\text {cris }}$ Fontaine defines two elements $\xi$ and $t$ where (in our picture) $\xi$ has as zeroset one point of valuation 1 whereas $t$ also has zeroes at its transforms under Frobenius (with valuations $p, p^{2}, \ldots$ ). If we choose $a>p^{-1}$ and $b \leq p-1$ the corresponding ring $A_{a, b}$ contains $A_{\text {cris }}$, and in it $\xi$ and $t$ generate the same ideal. This is useful because the étale- crystalline comparison theory gives information which is somehow inprecise up to a factor $t / \xi$. Also if $p>2$ we can still choose $A$ and $b$ such that $b \geq p a$, so the annulus has non trivial overlap with its Frobenius transform.

As an illustration let us consider the situation on tangent-spaces at the trivial objects, and there for rank one: The tangent space to representations is the étale $H^{1}\left(X_{\bar{K}}, \mathbb{Z}_{p}\right)$ and that to bundles with a usual connection the first crystalline cohomology $H_{\text {crys }}^{1}\left(X / A_{\text {inf }}(V)\right)$. For singular connections (which extends the previous notion of Higgs-bundle) we have to extend this by allowing elements in the first step of the Hodge-filtration to become divisible by $\xi^{-1}$, where $\xi$ denotes as before the wellknown element in Fontaine's theory. The crystalline comparison theorem shows that this is contained in $H^{1}\left(X_{\bar{K}}, \mathbb{Z}_{p}\right) \otimes A_{\text {inf }}(V)$, with the cokernel annihilated by $t / \xi$ but otherwise rather mysterious. If we pass to a certain $\tilde{A}(V)_{a, b} \supset A_{\text {inf }}(V)$ this cokernel (essentially) disappears and we obtain a good correspondence. Exponentiating this picture gives our result for "small" objects. We also present some ideas how to extend beyond these, and two examples to show that there are still difficult problems left. What is missing is an analogue of the exponential function from [6].

In the next sections we first generalise Berger's rings to general coefficients, explain the relation between Frobenius-modules and $p$-adic representations, and then (for curves) the link to bundles with singular connections.

It is an honor to dedicate this paper to the memory of Eckart Viehweg. 


\section{Berger's RINGS IN HIGHER DIMENSIONS}

Suppose $R$ is a $p$-adically complete torsionfree (commutative, with unit) algebra. We assume that $R[1 / p]$ is normal. Also in the following we also assume that $R$ is integral (otherwise everything will decompose into a product over irreducible components of $\operatorname{Spec}(R))$. We fix a dense open subset $U \subseteq \operatorname{Spec}(R[1 / p])$.

We denote by $\bar{R}$ the integral closure of $R$ in the maximal unramified covering of $U$, that is the union of all finite extensions $S$ contained in an algebraic closure of the fraction-field for which $S[1 / p]$ is étale over $R[1 / p]$ in all points of $U$. It admits a continuous action of the algebraic fundamental group (SGA1) $\pi_{1}(U)$. The Frobenius map is surjective on $\bar{R} / p \bar{R}$ (compare [7], lemma 3):

Suppose $x \in \bar{R}$ lies in a finite extension $S$ as above. Consider the extension $S^{\prime}$ of $S$ obtained by adjoining a root $y$ of the equation

$$
y^{p^{2}}-p y=x .
$$

It is finite (of degree $p^{2}$ ) over $S$ and thus $p$ lies in its Jacobson radical (the intersection of all maximal ideals). Furthermore the $y$-derivative of the above equation is

$$
p^{2} y^{p^{2}-1}-p=p\left(p y^{p^{2}-1}-1\right) .
$$

The second factor is invertible, and so is the whole derivative if we invert $p$. Thus $S^{\prime}[1 / p]$ is étale over $S[1 / p]$ and the inclusion $S \subset \bar{R}$ extends to a homomorphism which sends $y^{p}$ to a $p$-th root modulo $p$ of $x$.

Now define $\mathcal{R}(R)$ as the projective limit of $\bar{R} / p \bar{R}$ with Frobenius as transition maps. Equivalently an element of $\mathcal{R}(R)$ consists of an infinite sequence of elements $r_{n} \in \bar{R} / p \bar{R}$ (for $n \geq 0$ ) with

$$
r_{n}=r_{n+1}^{p} .
$$

We know that each such sequence lifts uniquely to such a sequence in the $p$-adic completion $\hat{\bar{R}}$. However the addition on such sequences is then no more defined componentwise. $\mathcal{R}(R)$ is a perfect ring of characteristic $p$, that is Frobenius on it is an isomorphism.

We denote by $\underline{p} \in \mathcal{R}(R)$ such a sequence with $r_{0}=p$ (it is unique up to multiplication by units), and by $\overline{1}$ a sequence with $r_{0}=1$ but $r_{1}$ is a primitive $p$-th root of unity. 


$$
A_{\text {inf }}(R)=W(\mathcal{R}(R))
$$

is the ring of Witt vectors. It is $p$-adically complete with

$$
A_{\text {inf }}(R) / p A_{\text {inf }}(R)=\mathcal{R}(R) .
$$

Any element of $A_{\text {inf }}(R)$ is an infinite sum

$$
\sum_{n \geq 0} p^{n}\left[r_{n, \bullet}\right]
$$

with Teichmüller representatives $\left[r_{n, \bullet}\right]$. The action of $\pi_{1}(U)$ on $\mathcal{R}(R)$ is continuous for the $(p,[\underline{p}])$-adic topology. There exists a homomorphism

$$
\theta: A_{\text {inf }}(R) \rightarrow \hat{\bar{R}}
$$

which sends a Teichmüller representative $\left[r_{\bullet}\right]$ to $r_{0}$. It is surjective with kernel generated by one element, for example by

$$
\xi=[\underline{p}]-p:
$$

It suffices to check that after reduction modulo $p$ ( $\xi$ clearly lies in the kernel). Then surjectivity follows from surjectivity of Frobenius on $\bar{R} / p \bar{R}$. Furthermore the kernel now consists of sequences $r_{\bullet} \in \mathcal{R}(R)$ with $r_{0}$ divisible by $p$. We have to show that each $r_{n} \in \hat{\bar{R}}$ is divisible by $p^{p^{-n}}$. For this approximate $r_{n}$ by an element of $\bar{R}$ which coincides with it modulo $p$. As the $p^{n}$-th power of this element is divisible by $p$ it lies itself in $p^{p^{-n}} \hat{\bar{R}}$.

For any positive rational number $a=m / n$ we denote by $\underline{p}^{a} \in \mathcal{R}(R)$ the $m$-th power of an $n$-th root of $p$. It is only well determined up to multiplication by a unit, but this will not matter in the sequel. For rational numbers $a, b, 0<a \leq b$, define (where \{\} denotes $p$-adically convergent series)

$$
\tilde{A}(R)_{a, b}=A_{\text {inf }}(R)\{X, Y\} /\left(X\left[\underline{p}^{a}\right]-p, Y p-\left[\underline{p}^{b}\right], X Y-\left[\underline{p}^{b-a}\right]\right),
$$

and

$$
A_{a, b}(R)=\tilde{A}_{a, b}(R)[1 / p] .
$$

Also

$$
\begin{gathered}
\tilde{A}(R)_{a, \infty}=A_{\text {inf }}(R)\{X\} /\left(X\left[\underline{p}^{a}\right]-p\right), \\
\tilde{A}(R)_{0, b}=A_{\text {inf }}(R)\{Y\} /\left(Y p-\left[\underline{p}^{b}\right]\right) .
\end{gathered}
$$


Lemma 1. a) The rings $\tilde{A}_{a, b}(R)$ are p-adically complete and separated, without p-torsion.

b) For $a \leq a^{\prime} \leq b^{\prime} \leq b$ we have inclusions (X maps to $\left[\underline{p}^{a^{\prime}-a}\right] X^{\prime}, Y$ to $\left[\underline{p}^{b-b^{\prime}}\right] Y^{\prime}$ )

$$
\tilde{A}(R)_{a, b} \subseteq \tilde{A}(R)_{a^{\prime}, b^{\prime}}, A(R)_{a, b} \subseteq A(R)_{a^{\prime} . b^{\prime}} .
$$

c) Under the same conditions

$$
\tilde{A}(R)_{a, b}=\tilde{A}(R)_{a, b^{\prime}} \cap \tilde{A}(R)_{a^{\prime}, b}
$$

(intersection in $\left.\tilde{A}(R)_{a^{\prime}, b^{\prime}}\right)$, and the same with $A^{\prime}$ 's.

Proof. The quotient $A_{\text {inf }}(R)\{X, Y\} /\left(X Y-\left[\underline{p}^{b-a}\right]\right)$ can be identified with the set of all $p$-adically convergent series

$$
\sum_{m \geq 0} a_{m} X^{m}+\sum_{n>0} b_{n} Y^{n}
$$

In this quotient the ideal generated by $X\left[\underline{p}^{a}\right]-p$ and $Y p-\left[\underline{p}^{b}\right]$ consists of all linear combinations (of $p$-adically convergent powerseries)

$$
\left(X\left[\underline{p}^{a}\right]-p\right) \sum_{m \geq 0} a_{m} X^{m}+\left(Y p-\left[\underline{p}^{b}\right]\right) \sum_{n \geq 0} b_{n} Y^{n} .
$$

As $[p]$ is a non zerodivisor in $A_{\text {inf }}(R) / p A_{\text {inf }}(R)$ this defines a topological direct sum, so the ideal is $p$-adically complete and thus closed. Hence the quotient is also $p$-adically complete and separated. Also one checks easily that it has no $p$-torsion.

Furthermore we derive an exact sequence

$$
(0) \rightarrow A_{\text {inf }}(R) \rightarrow \tilde{A}(R)_{0, b} \oplus \tilde{A}(R)_{a, \infty} \rightarrow \tilde{A}(R)_{a, b} \rightarrow(0),
$$

so to show assertion b) it suffices to treat the cases where $a=a^{\prime}=0$ or $b=b^{\prime}=$ $\infty$.

For the second consider a convergent series

$$
\sum_{m \geq 0} a_{m} X^{m}
$$

such that its image is divisible by $X^{\prime}\left[\underline{p}^{a^{\prime}}\right]-p$, that is

$$
\sum_{m} a_{m}\left[\underline{p}^{m\left(a^{\prime}-a\right)}\right] X^{\prime m}=\left(X^{\prime}\left[\underline{p}^{a^{\prime}}\right]-p\right) \sum_{n} b_{n} X^{\prime n} .
$$


This translates into

$$
\left[\underline{p}^{m\left(a^{\prime}-a\right)}\right] a_{m}=\left[\underline{p}^{a^{\prime}}\right] b_{m-1}-p b_{m} .
$$

As $p$ and $[p]$ form a regular sequence one derives by induction that $b_{m}$ is divisible by $\left[\underline{p}^{m\left(a^{\prime}-\bar{a}\right)}\right]$ and then that

$$
\sum_{m} a_{m} X^{m}
$$

is indeed divisible by

$$
X[\underline{p}]-p
$$

For the first inclusion assume that $Y^{\prime} p-\left[\underline{p}^{b^{\prime}}\right]$ divides $\sum_{n} b_{n}\left[\underline{p}^{n\left(b-b^{\prime}\right)}\right] Y^{\prime n}$, so

$$
\left[\underline{p}^{n\left(b-b^{\prime}\right)}\right] b_{n}=p c_{n-1}-\left[\underline{p}^{b^{\prime}}\right] c_{n} .
$$

We derive by induction over $r$ and (for fixed $r$ ) over $n$ that $c_{n}$ is divisible by

$$
\left[\underline{p}^{\operatorname{Min}\left(r b^{\prime},(n+1)\left(b-b^{\prime}\right)\right)}\right]
$$

and that

$$
\sum_{n} b_{n} Y^{m}
$$

is divisible by $Y p-\left[\underline{p}^{b}\right]$. c) also easily follows from the exact sequence above.

The rings $A(R)_{a, b}$ are $p$-adic Banach algebras (with the norms defined by $\tilde{A}$ ) and the inclusions have dense image (this is why we exclude $a^{\prime}=0$ ). We remark that the action of $\pi_{1}(U)$ on $\tilde{A}_{a, b}(R)$ is $p$-adically continuous if $b<\infty$.

We need a glueing result for "vectorbundles on overlapping annuli". For this we consider parameters

$$
0<a^{\prime} \leq a " \leq b^{\prime} \leq b^{\prime} \leq \infty,
$$

so we have an exact sequence

$$
(0) \rightarrow A(R)_{a^{\prime}, b^{\prime \prime}} \rightarrow A(R)_{a^{\prime}, b^{\prime}} \oplus A(R)_{a^{\prime \prime}, b^{\prime \prime}} \rightarrow A(R)_{a^{\prime \prime}, b^{\prime}} \rightarrow(0) .
$$

Suppose given finitely generated projective modules $M^{\prime}, M^{\prime \prime}$ over $A^{\prime}=A(R)_{a^{\prime}, b^{\prime}}$ respectively $A^{\prime \prime}=A(R)_{a ", b "}$, together with an isomorphism

$$
M \cong M^{\prime} \otimes_{A^{\prime}} A \cong M^{\prime \prime} \otimes_{A^{\prime \prime}} A
$$

over $A=A(R)_{a ", b^{\prime}}$. 
Lemma 2. Given these data the kernel $M^{*}$ of the natural difference map

$$
M^{\prime} \oplus M^{\prime \prime} \rightarrow M
$$

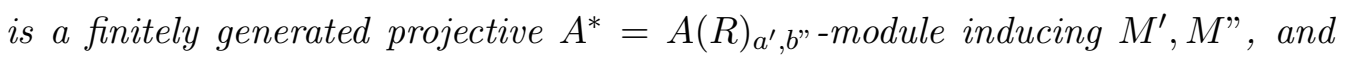
the isomorphism above.

Proof. After chosing systems of generators $M^{\prime}, M$ " and $M$ become Banach modules with norms well defined up to equivalence, and the inclusions $M^{\prime} \subseteq M$ and $M " \subseteq M$ have dense image. Choose a finite system of generators $m_{i}$ of $M$. Furthermore choose elements $m_{i}^{\prime} \in M^{\prime}$ with

$$
m_{i}-m_{i}^{\prime}=\sum_{j} f_{i j} m_{j}
$$

with $f_{i j} \subset p \tilde{A}(R)_{a ", b^{\prime}}$, and similarly elements $m_{i} " \in M "$. Then as

$$
\tilde{A}(R)_{a^{\prime \prime}, b^{\prime}}=\tilde{A}(R)_{a^{\prime}, b^{\prime}}+\tilde{A}(R)_{a^{\prime \prime}, b^{\prime \prime}}
$$

any $\tilde{A}(R)_{a^{\prime \prime}, b^{\prime}}$-linear combination of the $m_{i}$ can be written as a sum of an $\tilde{A}(R)_{a^{\prime}, b^{\prime}}$ linear combination of the $m_{i}^{\prime}$, an $\tilde{A}(R)_{a ", b}$ "-linear combination of the $m_{i}$ ", and a linear combination of the $m_{i}$ with coefficients in $p \tilde{A}(R)_{a^{\prime}, b^{\prime}}$. By $p$-adic iteration we can drop this last term.

If we define $M^{*}$ by our exact sequence above (i.e. as tuples of elements satisfying the glueing condition) we derive that the projections of $M^{*}$ to $M^{\prime}$ and $M$ " have dense image: For example given $m^{\prime} \in M^{\prime}$ approximate its image in $M$ by an $m " \in M$ " and write the difference $m^{\prime}-m$ " as a difference of elements in $M^{\prime}, M$ " with small norm. Correcting $m^{\prime}$ and $m$ " by these gives an element of $M^{*}$ approximating $m^{\prime}$.

It now follows easily that we can find finitely many elements $m_{i}^{*} \in M^{*}$ which generate $M^{\prime}$ as $A^{\prime}$-module and $M^{\prime \prime}$ as $A^{\prime \prime}$-module. If $N^{*}$ denotes the kernel of the resulting map

$$
A^{*, r} \rightarrow M^{*}
$$

$N^{*}$ is obtained by glueing the respective kernels $N^{\prime}, N^{\prime \prime}$ (over $A^{\prime}, A^{\prime \prime}$ ) using the same procedure as for $M^{*}$. To show that $M$ is a direct summand in $A^{*, r}$ we have to construct a section $s^{*}$ of the map $A^{*, r} \rightarrow M$. We know that we have such section $s^{\prime}, s^{\prime \prime}$ for $M^{\prime}$ and $M^{\prime \prime}$, . Their difference is an $A$-linear map $M \rightarrow N$. By the previous (applied to $\operatorname{Hom}(M, N)$ ) it can be written as a difference of 
maps in $\operatorname{Hom}\left(M^{\prime}, N^{\prime}\right)$ and $\operatorname{Hom}\left(M^{\prime \prime}, N^{\prime \prime}\right)$. Correcting $s^{\prime}$ and $s "$ by them gives a matching set of sections and thus the desired $s^{*}$.

Finally this implies that $M$ is finitely generated projective, and as $s^{*}$ induces $s^{\prime}$ and $s "$ it also follows that $M$ induces our gluing data. Uniqueness of $M$ is rather obvious.

The next topic is the introduction of Frobenius. The Frobenius automorphism of $\mathcal{R}(R)$ induces an automorphism on Witt-vectors and isomorphisms (as $[\underline{p}]$ is raised to its $p$-th power)

$$
\phi: A(R)_{a, b} \cong A(R)_{p a, p b} .
$$

If $b \geq p a$ we define an element of $A(R)_{a, b}$ to be $\phi$-invariant if $x=\phi(x)$ in $A(R)_{p a, b}$.

Lemma 3. Each Frobenius invariant element in $A(R)_{a, b}$ lies in $\mathbb{Q}_{p}$.

Proof. We may assume $b=p a$, and it suffices to consider Frobenius invariant elements in $\tilde{A}(R)_{a, b}$. We show that any such elements lies modulo $p$ in $\mathbb{F}_{p}$. The quotient

$$
\left.\tilde{A}(R)_{a, b} / p \tilde{A}(R)_{a, b}=\mathcal{R}(R)[X, Y] /\left(\left[\underline{p}^{a}\right] X,\left[\underline{p}^{b}\right], X Y_{\left[\underline{p}^{b-a}\right.}\right]\right)
$$

consists of polynomials

$$
f=\sum_{m>0} a_{m} X^{m}+\sum_{n \geq 0} b_{n} Y^{n}
$$

where the $a_{m}$ lie in $\mathcal{R}(R) /\left(\underline{p}^{a}\right)$ and the $b_{m}$ in $\mathcal{R}(R) /\left(\underline{p}^{b}\right)$. Frobenius invariance modulo $p$ means

$$
\phi\left(a_{m}\right)=\underline{p}^{m(p-1) a} a_{m}, \underline{p}^{n(p-1) b} \phi\left(b_{n}\right)=b_{n},
$$

where all equalities are modulo $\underline{p}^{b}$.

Now the first equalities imply that all $a_{m}$ vanish: They are represented by sequences $r_{n} \in \bar{R} / p \bar{R}$ such that $r_{n}=r_{n+1}^{p}$ and

$$
r_{n}^{p}=p^{n(p-1) a / p^{n}} r_{n}
$$

It follows that the quotients $r_{n} / p^{a / p^{n}}$ are integral over $\bar{R}$ and thus lie in $\bar{R}$, so the element $r_{n}$ is divisible by $\underline{p}^{a}$.

Next the equation

$$
b_{0}=b_{0}^{p}
$$


implies that $b_{0}$ defines a locally constant function from $\operatorname{Spec}\left(\mathcal{R}(R) /\left(\underline{p}^{b}\right)\right)$ to $\mathbb{F}_{p}$. It is constant if its domain of definition is connected. Otherwise the exists a non trivial idempotent in $\mathcal{R}(R) /\left(\underline{p}^{b}\right)$ and (as idempotents lift over nilpotents) a non trivial idempotent $e$ in $\bar{R} / p \bar{R}$. Thus there exists a finite $R$-algebra $S$, contained in the fraction-field of $R$, and a non trivial idempotent $e \in S / p S$. We claim that $e$ lifts to a non trivial idempotent in $S$, a contradiction as $S$ is an integral domain.

Lift $e$ to $S$, let $f=\left(e^{2}-e\right) / p \in S$ and let $F(f)=0$ denote a monic polynomial equation with coefficients in $R$ for $f$. The algebra

$$
S^{\prime}=R[u, v] /\left(u^{2}-u-p v, F(v)\right)
$$

is a finite free $R$-module and thus $p$-adically complete, $u$ defines an idempotent in $S^{\prime} / p S^{\prime}$ which lifts to an idempotent in $S^{\prime}$, and $S^{\prime}$ maps to $S$ sending $u$ to $e$ and $v$ to $f$. Thus we get a lift in $S$, and we have shown that $b_{0}$ lies in $\mathbb{F}_{p}$.

Finally the equation for the $b_{n}$ shows that they vanish (modulo $\left[\underline{p}^{b}\right]$ ) for positive $n$.

Under the same assumption on $a$ and $b$ we can consider Frobenius-modules. Such a module is a finitely generated projective $A(R)_{a, b}$ module $M$ together with an isomorphism

$$
\Phi: \phi^{*}(M) \cong M
$$

over $A(R)_{p a, b}$. By glueing this extends to a such a module $M_{a, b}$ over all $A(R)_{a, b}$, $0<a<b<\infty$. If $R$ is a complete discrete valuationring one of the main results of Kedlaya [11] say that such a module is the direct sum of submodules of constant slope, which are induced from $\mathbb{Q}_{p}$-vectorspaces with a Frobenius automorphism. If this automorphism can be chosen as the identity we call our module admissible. Equivalently the pair $(M, \Phi)$ if it is isomorphic to a direct sum of trivial modules $\left(A(R)_{a, b}, \phi\right)$.

For general $R$ recall that the Berkovich-spectrum of the Banach algebra $R[1 / p]$ consists of all multiplicative non archimedean seminorms which are $\leq 1$ on $R$, and coincide with the fixed $p$-adic norm on $\mathbb{Q}_{p}([2]$, Ch.1). Equivalently it consists of equivalence classes of morphism $R \rightarrow K$ into a completely valued $p$-adic field $K$. Such a morphism sends $R$ into the valuation-ring $V$ of $K$. One checks easily that Kedlaya's arguments in [11] still work for $V$. That is over $A(V)_{a, b}$ the pushout of a Frobenius module $M$ decomposes into components of fixed slopes. We call 
it admissible at the Berkovich point if all these slopes are zero, that is if the pushout to $A(V)_{a, b}$ becomes constant.

Recall also that the Berkovich spectrum is a compact topological space, with the topology defined by norms of functions $f \in R[1 / p]$. Each point has a fundamental system of (closed) neighborhoods (called "affinoid") which are Berkovich spectra of Banach algebras $R^{\prime}[1 / p]$. For example the norm of $f$ is $\leq 1$ on the Berkovich spectrum of $R[1 / p]\{f\}, \geq 1$ on that of $R[1 / p]\left\{f^{-1}\right\}$, etc.. The following is a variant of [7], th. 9 .

Proposition 4. Suppose $(M, \Phi)$ is a $\pi_{1}(U)$-equivariant Frobenius module over $A(R)_{a, b}, b \geq p a$. Then the set of points in the Berkovich spectrum where it is admissible is open. Furthermore each point in it has an "affinoid" neighbourhood given by an $R^{\prime}$ such that the pushout of $(M, \Phi)$ becomes trivial over $A\left(R^{\prime}\right)_{a, b}$.

Proof. We start with some general remarks on Berkovich spectra: A point in the Berkovich spectrum of $R[1 / p]$ is given by a homomorphism $x: R[1 / p] \rightarrow K$ into a field $K$ with a $p$-adic Banach norm. If $\mathfrak{p} \subset R[1 / p]$ denotes its kernel of $x$ (a prime ideal) we may assume that the fraction-field of $R[1 / p] / \mathfrak{p}$ is dense in $K$, so $K$ is a completion of that field. Denote by $V \subset K$ the valuation-ring.

The map $x: R \rightarrow V$ extends to $\bar{x}: \bar{R} \rightarrow \bar{V}$. This extension is not unique but two choices differ by the action of $\pi_{1}(U)$. More precisely if $D \subset \pi_{1}(U)$ denotes the joint stabiliser of an extension $\overline{\mathfrak{p}}$ of $\mathfrak{p}$ and an extension of the chosen $p$-adic norm on the fraction-field of $R / \mathfrak{p}$ then $D$ is a quotient of $\operatorname{Gal}(\bar{K} / K)$ which thus acts on $\bar{R}$ such that on extensions both actions coincide.

We also get a map $x: A(R)_{a, b} \rightarrow A(V)_{a, b}$. As $A_{\text {inf }}(V) \otimes_{A_{\text {inf }}(R)} A(R)_{a, b}$ is $p$-adically dense in $A(V)_{a, b}$ we can approximate any element of $A(V)_{a, b}$ by $A(R)_{a, b}$-linear combinations of Teichmüller representatives $[\underline{r}]$ with $\underline{r} \in \underline{p} \mathcal{R}(V)$. Such an $\underline{r}$ is given by a projective system of elements $r_{n} \in \bar{V} / p \bar{V}$. Each $r_{n}$ can be represented by an element of the valuation-ring $V^{\prime}$ of a finite extension $K^{\prime}$ of $K$. This extension is separable and generated by a single element. Approximating its minimal polynomial by a polynomial with values in the fraction-field of $R[1 / p] / \mathfrak{p}$ we may assume that the extension is defined by a separable polynomial over this field. Passing to a Berkovich-neighborhood we may assume that the polynomial 
has coefficients in $R[1 / p] / \mathfrak{p}$ (where the denominators are nonzero) and has invertible discriminant (same argument). So it defines a finite extension $R^{\prime}$ of $R$ which is étale after inverting $p$, and $R \rightarrow V$ extends uniquely to $x^{\prime}: R^{\prime} \rightarrow V^{\prime}$.

The element $r_{n}$ is a priori not in the image of this map. However we can assume that it lies in the image of $R^{\prime}[1 / p]$, by approximation. Assume that it is the image of an element $r / p^{s}$. As we know that $r_{n}$ has norm $<1$ we may pass to an open neighbourhood in the Berkovich spectrum of $R^{\prime}[1 / p]$ where the norm of $r / p^{s}$ is also $<1$. By uniqueness of $x^{\prime}$ this neighbourhood contains the preimage of a neighborhood of $x$. Also we can assume that $r$ is a unit in $R^{\prime}[1 / p] . p$-power roots of it lie in $\bar{R}$. All in all we obtain an element of $\mathcal{R}(R)$ whose image in $\mathcal{R}(V)$ is $\underline{p}$-adically close to $\underline{r}$. As $p$ divides in $A(V)_{a, b}$ a power of $[\underline{p}]$ we derive that any element of $A(V)_{a, b}$ can be approximated arbitrarily close in the $p$-adic topology by the image of $A(R)_{a, b}$, provided we pass to a Berkovich neighborhood.

What happens if we change our extension $\bar{R} \rightarrow \bar{V}$ by an element of $\pi_{1}(U)$ ? As $x^{\prime}$ is the unique Berkovich-point above $x$ such an element is the product of an element of the decomposition group $D$ of $x$ and an element which fixes the finite cover $R^{\prime}$. The second factor changes our element only by a $p$-adically small amount, and the first transforms the image via $\operatorname{Gal}(\bar{K} / K)$. Also we may apply thus procedure to finitely many elements $\underline{r} \in \mathcal{R}(V)$ at once, using a common extension $K^{\prime}$. Conclusion:

Suppose given finitely many elements $g_{1}, \ldots, g_{r} \in A(V)_{a, b}$, and $n>0$. Then there exists a neighbourhood of $x$ (defined by a new $R$ ) and elements $f_{1}, \ldots, f_{r} \in$ $A_{a, b}(R)$ such that for each extension $\bar{R} \rightarrow \bar{V}$ the image of the vector $\left(f_{1}, \ldots, f_{r}\right)$ is a $\operatorname{Gal}\left(\bar{K} / K\right.$-conjugate of $\left(g_{1}, \ldots, g_{r}\right)$ modulo $p^{n} \tilde{A}(V)_{a, b}$.

We also need that for an element $f \in A(R)_{a, b}$ with image in $A(V)_{a, b}$ of small $p$-adic norm (for any extension $\bar{R} \rightarrow \bar{V}$ ) the $p$-adic norm of $f$ becomes itself small if we pass to a Berkovich-neighborhood. We first show that for any rational $\epsilon>0$ and any $f \in \tilde{A}(R)_{a, b}$ whose image in $\tilde{A}(V)_{a . b}$ is divisible by $p,\left[p^{\epsilon}\right] f$ is divisible by $p$ in $\tilde{A}\left(R^{\prime}\right)_{a, b}$, for a neighbourhood of $x$ defined by $R^{\prime}$.

For this we use that

$$
\tilde{A}(R)_{a, b} /(p)=X \mathcal{R}(R)[X] /\left(\underline{p}^{a}\right) \oplus \mathcal{R}(R)[Y] /\left(\underline{p}^{b}\right) .
$$

So the reduction of $f$ modulo $p$ is given by finitely many coefficients $\left[\underline{r}_{i}\right]$ with elements $\underline{r}_{i} \in \mathcal{R}(R)$ whose image in $\mathcal{R}(V)$ is divisible by $p^{a}$ or $p^{b}$. Represent 
these by sequences of $p$-power roots (modulo $p$ ) in a finite generically étale $R$ algebra $S$. The assumption bounds their $p$-adic norm in all points of the Berkovich spectrum of $S[1 / p]$ mapping to $x$, so relaxing this bound as described by $\epsilon$ this still holds for a neighbourhood of $x$.

Repeating this argument shows that if the value of $f$ at $x$ is divisible by $p^{n}$ then this also holds for $\left[\underline{p}^{n \epsilon}\right] f$ in a neighbourhood. If $n \epsilon \leq a$ we derive that $f$ itself is divisible by $p^{n-1}$.

Now we apply these considerations to our problem. By assumption the $\operatorname{Gal}(\bar{K} / K)$ equivariant Frobenius module $M \otimes_{A(R)_{a, b}} A(V)_{a, b}$ has a basis consisting of Frobeniusinvariant elements. Lift this basis as well as the dual basis (for $M^{t}$ ) approximately to $M$ (the image under each extension $\bar{R} \rightarrow \bar{V}$ is close to a $\operatorname{Gal}(\bar{K} / K)$-conjugate of our basis or its dual). The pairing between $M$ and $M^{t}$ applied to these lifts gives a $h \times h$ matrix whose entries at $x$ (and all possible extensions of maps) are close to the identity. Replacing $R$ by a smaller neighbourhood we may assume that this matrix is congruent to the identity modulo $p \tilde{A}(R)_{a, b}$. This implies that our lifts form a basis for $M$, so $M$ is a free $A(R)_{a, b}$-module. Also we may assume that our basiselements are almost fixed by Frobenius (as this holds in $x$ ). So assume that we have a basis $m_{1}, \ldots, m_{h}$ of $M$ such that

$$
\Phi\left(m_{i}\right)-m_{i} \in p \sum_{j} \tilde{A}(R)_{p a, b} m_{j} .
$$

We correct them successively modulo $p$-powers to get a Frobenius-invariant basis. This is possible if the map

$$
\phi-i d: \tilde{A}(R)_{a, b} /(p) \rightarrow \tilde{A}(R)_{p a, b}
$$

is surjective. It sends a typical element

$$
\sum_{m>0} a_{m} X^{m}+\sum_{n \geq 0} b_{n} Y^{m}
$$

$\left(a_{m}\right.$ defined modulo $[\underline{p}]^{a}, b_{n}$ modulo $\left.[\underline{p}]^{b}\right)$ to

$$
\sum_{m>0}\left(\phi\left(a_{m}\right)-\underline{p}^{m(b-a)} a_{m}\right) X^{m}+\sum_{n \geq 0}\left(\underline{p}^{n(p-1) b} \phi\left(b_{n}\right)-b_{n}\right) Y^{n} .
$$

(all coefficients modulo $[\underline{p}]^{p a}$ )

To prove surjectivity of the map on coefficients note that on $\mathcal{R}(R) p^{r} \phi-i d$ is for $r>0$ surjective by an easy $p$-adic iteration. Furthermore as for any $\alpha<1, Y \in \bar{R}$ 
the equation $X^{p}-p^{\alpha} X=Y$ defines an étale cover of $\bar{R}[1 / p]$, the map $\phi-\underline{p}^{r} i d$ is surjective modulo each power $\underline{p}^{N}$.

Next we try to globalise these considerations. Suppose $V$ is a complete discrete valuation-ring, with perfect residue-field $k$ of characteristic $p>0$, and fractionfield $K$ of characteristic zero. We denote by $\pi$ a uniformiser of $V$. Also $V_{0}=W(k)$ are the Witt-vectors. Then $V$ is a finite totally ramified extension of $V_{0}$.

Suppose furthermore that $X$ is a proper semistable curve over $V$, with smooth generic fibre. Furthermore we assume that $D \subset X$ is a divisor which is finite étale over $V$, and we define our group $\pi_{1}$ as the fundamental group of $(X-D)_{K}$. That is we consider finite coverings which are unramified over $X_{K}-D_{K}$.

The condition on singularities means that $X$ can be covered by affines $U=$ $\operatorname{Spec}(R)$ with the algebra $R$ étale over $V_{0}[u, v]\left(u v-\pi^{n}\right)$ or over $V[u]$ (with $D$ defined by $u=0)$. This allows us to apply the theory of almost étale coverings to get a better grip on the rings $\bar{R}$ :

Namely choose a suitably ramified extension $V_{\infty}$ of $V$, for example the normalisation of $V$ in the field extension generated by all $p$-power roots of unity, or the extension obtained by adjoining all $p$-power roots of $\pi$. Then $\bar{R}$ contains the extension $R_{\infty}$ generated by $V_{\infty}$ and all roots of $u$, and is almost étale over it ([5], theorem 1.4). This means the following:

We can write $R_{\infty}$ as an increasing union

$$
R_{\infty}=\bigcup R_{n}
$$

of regular finite $R$-algebras $R_{n}$. If $S$ is a finite extension of $R$ unramified in characteristic zero the normalisation $S_{n}$ of $S \otimes_{R} R_{n}$ is a finite flat $R_{n}$-algebra. The discriminant of its trace-form divides for big $n$ a power of $p$ whose exponent converges to zero as $n \rightarrow \infty$. As a consequence the Galois-cohomology of $\bar{R} / R_{\infty}$ of any $\bar{R}$-module with continuous Galois-action is almost zero, that is annihilated by any positive fractional power of $p$. Similarly such a module is almost generated by its invariants. This allows us to reduce some statements about $\bar{R}$ to $R_{\infty}$ where we can do explicit calculations.

For the ring $\mathcal{R}(R)$ we have a similar result: The Frobenius is surjective $R_{\infty} / p R_{\infty}$ so we can form a projective limit $\mathcal{R}(R)_{\infty}$ and $\mathcal{R}(R)$ is almost étale over $\mathcal{R}(R)_{\infty}$ 
if we define "almost" with respect to the fractional powers of $[p]$. This then also applies to the rings $A_{\text {inf }}(R)$ and $\tilde{A}(R)_{a, b}, A(R)_{a, b}$. If $a>0$ note that $[p]$ becomes invertible in $A(R)_{a, b}$, so almost zero means zero.

Also the same reasoning works for the $p$-adic completion of $R$. One consequence of this theory is that $\pi_{1}$-equivariant almost modules over $\hat{\bar{R}}$ (or $\mathcal{R}(R)$, or $\tilde{A}(R)_{a, b}$ ) are the same category as over $R_{\infty}$ (with the corresponding smaller Galois-group). Also such modules then can be glued in the Zariski-topology, so can be defined over $X$ or better over the formal scheme $\hat{X}$. For example if $\mathbb{L}$ is a smooth $\mathbb{Q}_{p}$-sheaf on $X_{K}$ the tensorproducts $\mathbb{L} \otimes A(R)_{a, b}$ form a $\pi_{1}$-equivariant Frobenius module. Similar for $\mathbb{Z}_{p}$-sheaves and $\tilde{A}(R)_{a, b}$-modules. Theorem 4.9 in [5] says that under these circumstances the natural maps

$$
H^{i}\left(X_{K}, \mathbb{L}\right) \otimes_{\mathbb{Z}_{p}} \tilde{A}(V)_{a, b} \rightarrow H^{i}\left(\mathbb{L} \otimes_{\mathbb{Z}_{p}} \tilde{A}(R)_{a, b}\right)
$$

are almost isomorphisms. Thus our functor is fully faithful and the image is closed under extensions.

It is an open question which Frobenius modules arise from local systems. Of course it is necessary that they are pointwise admissible, but even then we only get a rigid étale $\mathbb{Q}_{p}$-sheaf which may not be algebraic. The latter is equivalent to admitting a $\mathbb{Z}_{p}$-lattice.

\section{BundLES With A Singular CONNECTION}

Frobenius modules should correspond to crystalline objects. We again assume that $X$ is a semistable proper curve over $\hat{\bar{V}}$. Furthermore we assume given a logarithmic lifting $X^{+}$to such a curve over $A_{\text {inf }}(V)$. Logarithmic means that the singularities of the lift are étale locally given by

$$
A_{\text {inf }}(V)[s, t] /\left(s t-[\underline{\pi}]^{\alpha}\right) .
$$

Such a lift always exists (by Grothendieck's classical argument as $A_{\text {inf }}(V)$ is complete in the $\xi$-adic topology) but it is not unique. Here $\xi$ is a generator of the kernel of $A_{\text {inf }}(V) \rightarrow \hat{\bar{V}}$, for example

$$
\xi=[\underline{p}]-p .
$$

For later purposes we need the notion of the logarithmic self-product of $X / A_{\text {inf }}$. This is a projective map

$$
X \times{ }^{\log } X \rightarrow X \times X
$$


which is an isomorphism away from the diagonal embedding of the discriminant locus of $X$. Recall that the singular locus of $X$ is the closed subset where $X$ is not smooth over $A_{\text {inf }}$. In local coordinates as above it is defined by the ideal $(s, t)$ which is also one of the Fitting-ideals of the relative (usual, not logarithmic) differentials. The discriminant locus is unramified over $A_{\text {inf }}$ and thus the disjoint union of sections over quotients $A_{\text {inf }} /[\underline{\pi}]^{\alpha}$. As the assertion is local around the singular locus we may assume that it consists only of one point.

If $Y \rightarrow X$ is an étale cover which is étale over $A_{\text {inf }}(V)[s, t] /\left(s t-[\underline{\pi}]^{\alpha}\right)$ consider the blowups of $Y \times{ }_{A_{\text {inf }}} Y$ in the ideals $\left(s_{1}, s_{2}\right)$ and $\left(t_{1}, t_{2}\right)$, with $s_{i}, t_{i}$ denoting the pullbacks of the coordinates from the factors. One checks that they are both isomorphic to $Y \times Y$ away from the selfproduct of the singular locus, and that the identity there extends to an isomorphism. The tensorproduct of the canonical ample line-bundles makes this into a projective scheme over $Y \times_{A_{\text {inf }}} Y$.

To descend to a projective scheme over $X \times_{A_{i n f}} X$ we have to check that the result is independant of the choice of coordinates $s, t$. As we already have canonical isomorphisms away from the singular locus it is enough to show that they extend, and this assertion is étale local in $X$. However one checks easily that in the strict henselisation of $X$ at the singular point two sets of coordinates $(s, t)$ and $\left(s^{\prime}, t^{\prime}\right)$ differ by first modifying by a unit $u\left(s^{\prime}=s u, t=t^{\prime} u\right)$ and (perhaps) by exchanging $s$ and $t$. The key fact which we use is that the discriminant $[\underline{\pi}]$ is a regular element in $A_{\text {inf }}$ :

The ideals $(s, t)$ and $\left(s^{\prime}, t^{\prime}\right)$ coincide. Thus either $s^{\prime}$ or $t^{\prime}$ generates this ideal modulo $s$, and we may assume that $t^{\prime}$ does. As $s$ divides $s^{\prime}$ after inverting either $s$ or $t^{\prime}$ the meromorphic function $s^{\prime} / s$ is regular outside the locus of $(s, t)$ which is generated by a regular sequence of length two. Thus $s$ divides $s^{\prime}$ and $t^{\prime}$ divides $t$, and because of $(s, t)=\left(s, t^{\prime}\right)$ the quotients are units.

Both types of coordinate changes extend to the blow-ups and induce isomorphims which respect the relatively ample bundle.

Finally we perform a similar operation on the diagonal embedding of $D$. If $D$ has local equation $\{u=0\}$ we blow-up $\left(u_{1}, u_{2}\right)$. The final result is a relatively projective

$$
X \times_{A_{\text {inf }}}^{\log } X \rightarrow X \times_{A_{i n f}} X
$$


such that $X$ embeds diagonally in it and is locally defined by one equation. This is an "exact immersion" (Kato). The corresponding conormal bundle is the sheaf of logarithmic differentials $\omega_{X}$.

There are also triple products etc: In local coordinates we blow up simultaneously $\left(s_{1}, s_{2}\right),\left(s_{1}, s_{3}\right),\left(s_{2}, s_{3}\right)$, that is we form the universal scheme where the $s_{i}$ become totally ordered under the relation of divisibility.

A vectorbundle with singular connection is a vectorbundle $\mathcal{E}$ on $X^{+} \otimes_{A_{\text {inf }}(V)}$ $\tilde{A}(V)_{a, b}$ together with a

$$
\nabla: \mathcal{E} \rightarrow \mathcal{E} \otimes_{A_{i n f}(V)} \xi^{-1} \omega_{X^{+}}
$$

satisfying the usual connection rule, and such that the Taylor-series converge $p$ adically. That means on the smooth locus that for a local coordinate $t$ and a local section $e$ of $\mathcal{E}$ the sequence $\xi^{n} \nabla(\partial / \partial t)^{n}(e) / n$ ! converges $p$-adically to zero. A variant also applies to singular points. Equivalently we may consider the $p$ adic formal scheme $\hat{D}_{\xi}^{(2)}\left(X^{+}\right)$over $A_{\text {inf }}(V)$ obtained by adjoining $\mathcal{I}_{\Delta} / \xi$ to the algebra of the logarithmic selfproduct $X^{+} x^{\log } X^{+}$. A singular connection is an isomorphism between the two pullbacks of $\mathcal{E}$ which satisfies transitivity on the triple product.

We also switch freely between formal vectorbundles and algebraic bundles:

Lemma 5. The restriction of vectorbundles on $X^{+}$to those on $\hat{X}^{+}$is an equivalence of categories, over bases $A_{\text {inf }}(V), A_{\text {cris }}(V)$, or $\tilde{A}_{a, b}(V)$. The same holds for bundles with singular connections.

Proof. If $\mathcal{E}$ is a formal vectorbundle its reduction modulo $p$ is algebraic and induced from a vectorbundle over a noetherian base. Tensoring with a suitable very ample line-bundle $\mathcal{L}$ we may assume that the higher direct images of $\mathcal{E} \otimes \mathcal{L} /(p)$ vanish fibrewise, and the zeroe'th direct image generates this bundle. It follows that we can lift sections and that $\mathcal{E}$ is the quotient of a direct sum of copies of $\mathcal{L}^{-1}$. It thus defines a $p$-adic sequence of points in a Quot-scheme which has an algebraic limit. Thus $\mathcal{E}$ is algebraic. For maps the argument is similar, again writing $\mathcal{E}$ as quotient of an algebraic bundle.

Finally for singular connections there exists an exact sequence of bundles

$$
0 \rightarrow \mathcal{E} \otimes \xi^{-1} \omega_{X} \rightarrow J_{\xi}^{(2)}(\mathcal{E}) \rightarrow \mathcal{E} \rightarrow 0
$$


and singular connections correspond to splittings.

A logarithmic connection corresponds to an isomorphism between the two pullbacks of $\mathcal{E}$ to $\hat{D}_{\xi}^{(2)}\left(X^{+}\right)$which satisfies the usual cocycle relation on the triple product $D_{\xi}^{(3)}\left(X^{+}\right)$. Equivalently the logarithmic selfproducts form a groupoid of formal schemes which operates on $\hat{X}^{+}$, and $\mathcal{E}$ is a bundle on the quotient. Or (a third description) the dual $\mathcal{D}_{\xi}$ of the direct image (under the first projection to $X^{+}$) of the structuresheaf of $D^{(2)}\left(X^{+}\right)$is a sheaf of topological noncommutative algebras on $X^{+}$and an $\mathcal{O}_{X^{+}}$-bimodule, and a singular connection on $\mathcal{E}$ is the same as a continuous $\mathcal{D}_{\xi}$-operation. $\mathcal{D}_{\xi}$ is a projective limit of locally free $\mathcal{O}_{X^{+}}$-modules, and locally on the smooth locus it is isomorphic to the algebra of power-series in divided powers $(\xi \partial / \partial t)^{n} / n$ !, for any local coordinate $t$. At the double points we have to replace these by $\xi^{n} D(D-1) \ldots(D-n+1) / n$ ! with $D=t \partial / \partial t$ for a log-coordinate $t$.

Assume given a non constant map $X \rightarrow Y$ between two semistable curves over $\bar{V}$. It preserves the $\log$ structure, and locally on open subsets $\hat{U} \subset \hat{X}$ we can extend to a logarithmic map $X^{+} \rightarrow Y^{+}$for lifts over $A_{\text {inf }}(V)$. Two such extensions define a $\hat{U} \rightarrow D_{\xi}^{(2)}(Y)$ and we get a map on stack-quotients (under groupoid-actions)

$$
\hat{X}^{+} / \hat{D}_{\xi}^{(2)}\left(X^{+}\right) \rightarrow \hat{Y}^{+} / \hat{D}_{\xi}^{(2)}\left(Y^{+}\right) .
$$

Stack-quotient means that the domain of definition $\hat{X}^{+}$has to be replaced by a Zariski cover, with the pullback groupoid.

Thus if $\mathcal{E}$ on $\hat{Y}^{+}$admits an action of $D_{\xi}^{(2)}\left(Y^{+}\right)$we get a welldefined pullback on $\hat{X}^{+}$. Applied to $X=Y$ we especially derive that the category of vectorbundles with singular connections does not depend on the choice of the lift $X^{+}$.

The singular connection $\nabla$ defines a $\pi_{1}$-equivariant module, as follows: Suppose $\operatorname{Spec}(R) \subset X$ is a small affine which we lift to an affine $\operatorname{Spec}\left(R^{+}\right) \subset X^{+}$. Choose an $A_{\text {inf }}(V)$-linear lift $f$

$$
R^{+} \rightarrow A_{\text {inf }}(R)
$$

of $R^{+} \rightarrow \hat{\bar{R}}$ und the pushforward by it of $\mathcal{E}$. For two different lifts the connection gives an isomorphism between the two pushforwards, via the Taylor-series

$$
\sum_{n} \nabla\left(\partial_{u}\right)^{n}(e)\left(f_{2}(u)-f_{1}(u)\right)^{n} / n !
$$


Here $u$ denotes a local coordinate, $e$ a local section of $\mathcal{E}$. This series converges $p$-adically so we get the desired isomorphism. Note that $f_{2}(u)-f_{1}(u)$ is divisible by $\xi$ so the terms of the series have no denominators $\xi$. An example (if $b<\infty$ ) is given by the trivial bundle $\mathcal{O}_{X^{+}}$with its trivial connection: $\partial_{u}^{n}$ is divisible by $n$ ! (because the usual Taylor-series converges) and $p$ divides $\left[p^{b}\right]$ and thus also a sufficiently high power of $[p]-p$. This reasoning also implies that the $e$ 's for which we have convergence form an $R^{+}$-submodule.

One possible assumption which implies convergence is that $\mathcal{E}, \nabla$ is locally trivial modulo a positive $p$-power $p^{s}$, that is locally $\mathcal{E}$ has a basis annihilated modulo $p^{s}$ by $\nabla$, or equivalently locally

$$
\mathcal{E} \cong \mathcal{O}_{X}^{r}
$$

and

$$
\nabla=d+\alpha d u
$$

where $\alpha$ is a $r \times r$-matrix with entries in $p^{s} \xi^{-1} R$. We claim that the series then converges if $b \leq p-1$ and if in addition either $p>2$ or $s \geq 2$ :

Applied to a basis-element $e$ we have to sum

$$
\partial_{u}^{n_{1}} \alpha \partial_{u}^{n_{2}} \alpha \ldots \partial_{u}^{n_{j}} \alpha(e) \xi^{n+j} /(n+j) !
$$

over all tupes $\left(n_{1}, \ldots, n_{j}\right)$ of non-negative integers (with $n$ their sum).

All the partials are divisible by $n_{i}$ !, the $\xi \alpha$ by $p^{s}$, and $\xi^{p-1}$ by $p$. If we ignore the partials we get as $p$-power (where $\sigma(n+j$ ) denotes the sum of the digits of $n+j$ in $p$-adic numbers)

$$
j s+[n /(p-1)]-(n+j) /(p-1)+\sigma(n+j) /(p-1) .
$$

This is the largest integer below or equal

$$
j s-(j-\sigma(n+j)) /(p-1) \geq s+(j-1)(s-1 /(p-1)) .
$$

Thus we obtain a $p$-power which is at least $p^{s}$ and converges to infinity if $j \rightarrow \infty$. If $j$ remains bounded but some of the $n_{j} \rightarrow \infty$ we get additional unbounded $p$ powers from the factors $n_{j}$ ! which we have not used yet. Thus the series converges and all terms are divisible by $p^{s}$.

Also we obtain under these conditions a Frobenius pullback for bundles trivial modulo $p^{s}$, which sends bundles with coefficients in $\tilde{A}_{a, b / p}$ to those with coefficients $\tilde{A}_{p a, b}$ : 
In $\tilde{A}(V)_{p a, b}$

$$
\xi=[\underline{p}]-p=[\underline{p}](1-p /[\underline{p}])
$$

has the same divisor as $[\underline{p}]$ and divides $p$ (as $p a>1$ ). Thus two different local Frobenius-lifts coincide modulo $\xi$ and the Taylor-series comparing the two pullbacks coincides.

In the following we have to pass to the almost category, that is we consider almost modules over $R^{+} \otimes_{A_{\text {inf }}(V)} \tilde{A}_{a, b}(R)$ with a singular connections, and almost modules over $\tilde{\mathcal{A}}_{a, b}(R)$.

Another consequence of $b \leq p-1$ is that $\xi^{p-1}$ is divisible by $p$ in $\tilde{A}(R)_{a, b}$ and thus the ring $A_{\text {cris }}(R)$ maps to $\tilde{A}_{a, b}$. In fact each power $\xi^{n}$ is in $\tilde{A}_{a, b}$ divisible by $(n+1)$ !. The crystalline comparison theorem ([5], cor.5.4) defines an almost map

$$
H_{D R}^{1}\left(X^{+}, \mathcal{O}\right) \otimes_{A_{\text {inf }}(V)} A_{\text {cris }}(V) \rightarrow H_{\text {et }}^{1}\left(X_{\bar{K}}, \mathbb{Z}_{p}\right) \otimes_{\mathbb{Z}_{p}} A_{\text {cris }}(V)
$$

which respects Poincaré-duality up to a factor $t$ and thus has an inverse up to this factor. Furthermore the first step $\Gamma\left(X^{+}, \omega^{1}\right)$ of the Hodge-filtration has image contained in the tensorproduct with $F^{1}\left(A_{\text {cris }}(V)\right.$. If we base extend to $\tilde{A}(V)_{a, b}$ the image is then contained in the ideal generated by divided powers $\xi^{n} / n$ ! with $n>0$. Thus the image is divisible by $\xi$.

Geometrically this map has the following interpretation: Consider extensions

$$
(0) \rightarrow \mathcal{O} \rightarrow \mathcal{E} \rightarrow \mathcal{O} \rightarrow(0)
$$

in the category of vectorbundles on $X^{+}$with singular connections, with coefficients in $\tilde{A}_{a, b}$. Then the Taylor-series converges for such extensions: In the notation above $\alpha$ becomes unipotent with square zero, and we have to sum

$$
\partial_{u}^{n} \alpha \xi^{n+1} /(n+1) !
$$

which is divisible by $\alpha \xi^{n} /(n+1)$ and thus converges $p$-adically to zero..

So we get an almost $\pi_{1}$-equivariant module over the $\tilde{A}(R)_{a, b}$. This module is defined by an extension of trivial étale $\mathbb{Z}_{p}$-sheaves.

If we multiply the Poincaré duality product on $H_{D R}^{1}\left(X^{+}\right) \otimes_{A_{\text {inf }}(V)} \tilde{A}(V)_{a, b}$ by $\xi$ we get a non-degenerate product on the space describing such extensions $\mathcal{E}$ with singular connections. As our map respects dualities up to a factor $t / \xi$ there exists an inverse up to $t / \xi$, so this factor then annihilates the kernel and cokernel. 
We claim that $t / \xi$ divides $p^{2}$ (in $\left.\tilde{A}(R)_{a, b}\right)$ provided $a>1 / p$ : It is known that $t / \xi$ is up to a unit equal to $1-\left[\underline{\zeta}_{p}\right]$, where the second term denotes the Teichmüller representative of a sequence of $p$-power roots of a primitive $p$-th root of unity. As it is equal (in $\mathcal{R}(R))$ to 1 modulo $\underline{p}^{1 /(p-1)}$ the image of $1-\left[\underline{\zeta}_{p}\right]$ in the Witt vectors over $\mathcal{R}(R) /\left(\underline{p}^{1 /(p-1)}\right)$ vanishes. This means that in $A_{\text {inf }}(R)$ we have an expansion

$$
1-\left[\underline{\zeta}_{p}\right]=\sum_{n} p^{n}\left[z_{n}\right]
$$

with $z_{n} \in \mathcal{R}(R)$ divisible by $\underline{p}^{p^{-n} /(p-1)}$. Also the leading term becomes a unit if we divide by this $\underline{p}$-power. Hence

$$
1-\left[\underline{\zeta}_{p}\right]=\left[\underline{p}^{1 /(p-1)}\right]\left[u_{0}\right]\left(1+\sum_{n>0}\left[\underline{p}^{n a-\left(1-p^{-n}\right) /(p-1)}\right]\left[w_{n}\right] X^{n}\right)
$$

with $u_{0}$ a unit, and this is up to a unit equal to $\left[\underline{p}^{1 /(p-1)}\right]$ in $\tilde{A}(R)_{a, b}$ if $a>1 / p$. It then also divides $p^{2}$.

The de Rham cohomology with coefficients in $\xi^{-1} \Omega$ defines a cohomological functor on vectorbundles with singular connection. The functor to $\pi_{1}$-equivariant modules induces maps on cohomology in degrees zero and one (defined by extensions). To circumvent the problem of treating degree two we pass to the logarithmic context, that is assume that the divisor at infinity is nonempty. This has the advantage that various second cohomology groups will vanish.

Our formulas work for almost modules modulo a power $p^{n}$, and for bundles modulo $p^{n}$ with singular connections. They show that there exists fixed numbers $s, t$ such that a bundle modulo $p^{n}$ with singular connection trivial modulo $p^{s}$ defines a $\pi_{1}$-equivariant almost module modulo $p^{n}$. As the relevant cohomology vanishes in degree two (for bundles with singular connections because they are unipotent modulo $p$ ) we can always lift bundles modulo $p^{n}$ to bundles modulo $p^{n+1}$, and the same holds for equivariant almost modules.

We have seen that any extension of trivial bundles defines an extension of almost modules. As the corresponding map on first cohomology groups has kernel and cokernel annihilated by a fixed power $p^{t}$ it follows that if two singular extensions modulo $p^{n}$ induce isomorphic extensions of almost modules then they are already isomorphic modulo $p^{n-t}$, and that any extension modulo $p^{n}$ of trivial almost modules which is trivial modulo $p^{r+t}$ (for some $r \geq 0$ ) is induced from an extension (trivial modulo $p^{r}$ ) of trivial bundles modulo $p^{n}$. We derive from that: 
Proposition 6. Suppose $n \geq s+t+1$. Then any $\pi_{1}$-equivariant almost module modulo $p^{n}$ trivial modulo $p^{s+t}$ is induced from a bundle (modulo $p^{n}$ ) with singular connection trivial modulo $p^{s}$. Furthermore for bundles trivial modulo $p^{s+t+1}$ any map of such modules is induced modulo $p^{n-t}$ by a map of bundles modulo $p^{n-t}$, unique modulo $p^{n-s-t}$.

Proof. We use induction over $n$. The start $n=s+t$ is trivial. Assume the result holds for $n-1$. Given a representation modulo $p^{n}$, trivial modulo $p^{s+t}$, its reduction modulo $p^{n-1}$ comes from a bundle modulo $p^{n-1}$ which is trivial modulo $p^{s}$. It lifts modulo $p^{n}$ (the relevant second de Rham cohomology vanishes) and induces a new $\pi_{1}$-equivariant module modulo $p^{n}$. It differs from the original module by a class in $H^{1}$ which corresponds to an extension trivial modulo $p^{n-1}$ of of trivial almost modules modulo $p^{n}$. By the previous it is induced by an extension of trivial bundles modulo $p^{n}$, trivial modulo $p^{n-1-t}$. Use this extension to modify our lift, and we get the first assertion.

For maps we proceed in the same way: Given a map modulo $p^{n}$ first consider its reduction modulo $p^{n-1-t}$ which is induced by a map of bundles modulo $p^{n-1-t}$, by induction. Trying to lift this map modulo $p^{n}$. The obstruction to this is an extension of trivial bundles modulo $p^{n}$, trivial modulo $p^{n-1-t}$. Its image on almost modules vanishes modulo $p^{n}$, so the class itself vanishes modulo $p^{n-t}$. Thus we may lift modulo $p^{n-t}$ our map on bundles. The lift may not induce the desired map on almost modules, but the two differ by a map modulo $p^{n-t}$ which is trivial modulo $p^{n-1-t}$, i.e. an endomorphism of the trivial almost module modulo $p$. It is induced from a map of trivial bundles modulo $p$ or by a map of bundles modulo $p^{n-t}$ which is trivial modulo $p^{n-1-t}$.

Passing to the limit $n \rightarrow \infty$ we obtain that almost modules trivial modulo $p^{s+t}$ are induced from bundles with singular connections trivial modulo $p^{s}$, and homomorphisms correspond. Also the Frobenius on representations induces an isomorphism of Frobenius pullbacks which map the pushouts to $\tilde{A}(V)_{a, b / p}$ to those to $\tilde{A}(V)_{p a, b}$.

Finally in the general case where the divisor $D$ is allowed to by empty we choose two such fiberwise divisors $D^{\prime}$ and $D^{\prime \prime}$ and use that the singular connections without poles are the intersection of singular connections with poles in $D^{\prime}$ and 
singular connections with poles in $D^{\prime \prime}$, the intersection taking place in connections with poles in $D^{\prime}+D^{\prime \prime}$. Also there is an analogue for almost modules.

For arbitrary $\mathbb{Z}_{p}$ representations of the fundamental group, not necessarily close to trivial, we can find a Galois cover (defined over $\hat{\bar{V}}$ )

$$
Y \rightarrow X
$$

such that the representation becomes sufficiently close to the identity on $\pi_{1}(Y)$. Here $Y$ is semistable, the generic fibre is a $G$-cover, and we may assume that the map is stable (as defined by Kontsevich-Manin). We then get a bundle with singular connection on any lift $Y^{+}$of $Y$ to $A_{\text {inf }}(V)$ and this lift is $G$-equivariant for the twisted action on $\hat{Y}^{+} / D_{\xi}^{(2)}\left(Y^{+}\right)$. So all in all the bundle is equivariant under a groupoid which is an extension of $G$ by $D_{\xi}^{(2)}\left(Y^{+}\right)$. However I do not know how to relate these data to bundles on $X^{+}$.

\section{EXAMPLES}

a) Consider $X=\mathbb{P}^{1}$ with $D=\{0, \infty\}$. We consider representations and bundles of rank one. The geometric fundamental group is $\hat{\mathbb{Z}}(1)$ so representations on $\mathbb{Q}_{p}$ are non canonically identified with $\mathbb{Z}_{p}^{*}$. For line-bundles with singular connections one gets for bundle always the trivial bundle $\mathcal{O}_{X}$, with connection

$$
\nabla_{a}=d+a d T / T
$$

with $a \in \xi^{-1} A(V)_{a, b}$. The action of Frobenius can be defined without convergence problems as $X$ admits the Frobenius-lift given by $T^{p}$ and it sends $\nabla_{a}$ to $\nabla_{p \phi(a)}$. The fixed-points are $\mathbb{Q}_{p} t^{-1}$. There is a natural map from representations to Frobenius-bundles with singular connection

$$
\operatorname{Hom}\left(\mathbb{Z}_{p}(1), \mathbb{Z}_{p}^{*}\right) \stackrel{\log }{\rightarrow} \operatorname{Hom}\left(\mathbb{Z}_{p}(1), \mathbb{Q}_{p}\right)=\mathbb{Q}_{p} t^{-1}
$$

which on small representations coincides with our general construction, and which is neither injective nor surjective. This example suggests that one should impose some conditions on the monodromy around $D$, as in [6].

b) Assume $X$ has good reduction and $D$ is empty. Line-bundles with a connection are classified by the $A_{\text {inf }}(V)$-points of the universal vectorextension $E$ of the Jacobian $J$, which lies in an exact sequence

$$
0 \rightarrow \Omega_{X} \rightarrow E \rightarrow J \rightarrow 0
$$


with $\Omega_{X}$ the vectorgroup given by the differentials on $X$. It admits a Frobeniuslift over $A_{\text {inf }}(V)$ (after lifting $X$ ). Singular connections correspond to baseextension by embedding $\Omega_{X}$ into its tensor-product with $\xi^{-1} A(V)_{a, b}$. A natural Frobenius-lift can be defined for some such extensions but not all of them. Representations of the geometric fundamental-group into $\mathbb{Z}_{p}^{*}$ are given by homomorphisms defined on the Tate-module $T_{p}(J)$. Here the torsion-elements correspond to the $(p-1)$-torsion in $E$ and are all elements of $E\left(A(V)_{a, b}\right)$.

On the other hand representations with values in $1+p \mathbb{Z}_{p}$ correspond via the exponential to representations into $p \mathbb{Z}_{p}$. The crystalline comparison theorem defines an inclusion

$$
T_{p}(J) \otimes A_{\text {crys }}(V) \rightarrow \operatorname{Lie}\left(E / A_{\text {crys }}(V)\right)
$$

with cokernel annihilated by $t$. It induces an isomorphism

$$
T_{p}(J)(-1) \otimes A(V)_{a, b} \cong\left(\operatorname{Lie}\left(E / A_{i n f}(V)+\xi^{-1} \Omega_{X}\right) \otimes A(V)_{a, b} .\right.
$$

The representations with values in $p \mathbb{Z}_{p}$ are naturally isomorphic to $p T_{p}(J)(-1)$. Via the exponential they correspond to certain elements in the pushout of the universal vectorextension. Frobenius-lifts exist on a suitable subgroup (the image under the exponential) of this pushout as well as on the original vectorextension. They combine to give a partial Frobenius with fixed-points the desired representations.

c) Assume $\mathcal{E}$ is a filtered Frobenius-crystal on $X$. It extends naturally to any lift $X^{+}$to $A_{\text {cris }}$. It is associated to a $p$-adic local system $\mathbb{L}$ if

$$
\mathcal{E}\left(B_{\text {cris }}(R)\right)=\mathbb{L} \otimes B_{\text {cris }}(R)
$$

for affines $\operatorname{Spec}(R) \subset X$ (see [4]). With the induced product filtration

$$
F^{0}\left(\mathcal{E} \otimes_{A_{\text {cris }}(V)} B_{\text {cris }}(V)\right.
$$

is a bundle with singular connection and it corresponds to $\mathbb{L}$.

\section{REFERENCES}

[1] V.G.Berkovich, Spectral theory and analytic geometry over non-archimedean fields, American Mathematic Society, Providence 1990

[2] L.Berger, Représentations p-adiques et équations differentielles, Inv. math. 148 (2002), 219284 
[3] S.Bosch, U.Güntzer, R.Remmert, Non archimedean analysis. A systematic approach to rigid analytic geometry, Grundlehren 261, Springer-Verlag, Berlin 1984

[4] G.Faltings, Crystalline cohomology and p-adic Galois representations, in:AlgebraicAnalysis, GeometryandNumberTheory (J.Igusa, ed.), Johns Hopkins Univ. Press, Baltimore 1989, 25-80

[5] G.Faltings, Almost étale extensions, Astérisque 279 (2002), 185-270

[6] G.Faltings, A p-adic Simpson correspondence, Advances in Mathematics 198 (2005), 847862

[7] G.Faltings, Coverings of p-adic period domains, J.reine angew. Math. 643 (2010), 111-139

[8] J.M.Fontaine, Modules galoisiennes, modules filtrés et anneaux de Barsotti-Tate, Astérisque 65 (1979), 3-80

[9] J.M.Fontaine, Le corps des périodes p-adiques, Astérisque 223 (1994), 59-102

[10] A.J. de Jong, Étale fundamental groups of non-Archimedean analytic spaces, Comp. math. 97 (1995), 89-118

[11] K.S.Kedlaya, A p-adic local monodromy theorem, Ann. of Math. 160 (2004), 93-184

[12] B.Mazur, W.Messing, Universal extensions and one dimensional crystalline cohomology, Springer lecture Notes 370 (1974)

\section{Gerd Faltings}

Max-Planck-Institut für Mathematik

Vivatsgasse 7

53111 Bonn, Germany

E-mail: faltings@mpim-bonn.mpg.de 